\title{
Endothelial dysfunction and increased platelet reactivity in patients with acute coronary syndrome and undiagnosed COPD: insights into the SCAP trial
}

\begin{abstract}
To the Editor:
Unknown/undiagnosed chronic obstructive pulmonary disease (UCOPD) frequently coexists and negatively impacts in the short- and long-term prognosis of patients with coronary artery disease (CAD) treated with percutaneous coronary intervention (PCI) [1]. In the Screening for COPD in Acute coronary syndromes Patients (SCAP) trial [2], we recently showed that COPD is frequently undiagnosed in ccute coronary syndrome (ACS) patients with smoking history. Furthermore, we found that a simple screening procedure including peak expiratory flow measurement and respiratory health status questionnaire (RHSQ) might be administered before hospital discharge to discriminate those at risk of UCOPD. In the SCAP study, it was pre-specified that an ancillary analysis aimed to clarify if UCOPD was related to abnormalities in platelet reactivity and endothelial function.

Inclusion and exclusion criteria of the SCAP trial have been previously reported [2]. Briefly, current or former smoking ( $\geqslant 10$ pack-years) patients admitted to hospital with a diagnosis of ACS were enrolled. ACS diagnosis was based according to the criteria of current guidelines [3], as well as cardiovascular management and pharmacological therapy $[3,4]$. Exclusion criteria were as follows: previous diagnosis of COPD and/or asthma, known pulmonary disease other than COPD, ongoing pneumonia, ongoing heart failure, documented or suspicion of malignant disease, life expectancy $<1$ year, and recent thoracic trauma. The P2Y12 inhibitor (ticagrelor versus clopidogrel versus prasugrel) was selected according to the physician's discretion. After 2 months from the time of discharge, patients underwent spirometry. COPD was defined by the presence of irreversible airflow obstruction (forced expiratory volume in $1 \mathrm{~s} /$ forced vital capacity $<70 \%$ post-bronchodilator) [4]. The work was approved by the institutional ethics committee, and informed written consent was obtained from each subject. This study is registered at ClinicalTrials.gov (number: NCT02324660). A venous blood sample was collected from all patients at the time of the enrolment, which occurred at hospital discharge. Of note, the blood sample was collected when the diagnosis of COPD was still unknown. All patients underwent blood sampling early morning (08:00-10:00 $\mathrm{h}$ ), after at least $30 \mathrm{~min}$ of rest and $2 \mathrm{~h}$ of fasting. Blood sample was obtained from an antecubital vein using a 21-gauge needle. The specific analyses for the present study were: 1) endothelial dysfunction assay, assessed by the rate of apoptosis in human umbilical vein endothelial cells (HUVECs) after 48-h incubation with $20 \%$ serum from patients, as previously reported $[5,6] ; 2$ ) on-treatment platelet reactivity measured with light transmittance aggregometry after arachidonic acid and adenosine diphosphate stimulus [7]. Continuous data were tested for normal distribution with the Kolmogorov-Smirnov test. Normally distributed values were presented as mean \pm SD and compared by t-test and one-way ANOVA. Otherwise, median (interquartile range), Mann-Whitney $U$ - and Kruskal-Wallis tests were used. Categorical variables were summarised in terms of number and percentages and were compared by using two-sided Fisher's exact test. The association between all variables are listed in table 1. UCOPD and the rate of apoptosis was assessed by logistic regression models. Variables with a probability value $<0.1$ in the univariate analysis (age, diabetes, UCOPD, ticagrelor) and potential confounding factors (sex, clinical presentation, current smoking) were then entered into a multivariable analysis to identify the independent
\end{abstract}

@ERSpublications

Endothelial dysfunction and increased platelet reactivity in acute coronary syndrome with unknown/ undiagnosed COPD http://ow.ly/SVEg30eExbK

Cite this article as: Pavasini R, Vieceli dalla Sega F, Gallo F, et al. Endothelial dysfunction and increased platelet reactivity in patients with acute coronary syndrome and undiagnosed COPD: insights into the SCAP trial. Eur Respir J 2017; 50: 1701183 [https://doi.org/10.1183/13993003.01183-2017]. 
TABLE 1 Characteristics of the study population

\begin{tabular}{|c|c|c|c|}
\hline & No COPD $(n=98)$ & UCOPD (n=39) & p-value \\
\hline Age years & $63 \pm 11$ & $69 \pm 8$ & $<0.01$ \\
\hline Male n (\%) & 83 (85) & 32 (82) & 0.8 \\
\hline BMI $\mathrm{k} \cdot \mathrm{m}^{-2}$ & $28 \pm 4$ & $27 \pm 4$ & 0.2 \\
\hline Diabetes n (\%) & $21(21)$ & $11(28)$ & 0.5 \\
\hline Hypertension n (\%) & $67(68)$ & $25(64)$ & 0.7 \\
\hline Hyperlipidaemia n (\%) & $55(56)$ & $17(44)$ & 0.2 \\
\hline Current smoker n (\%) & $44(45)$ & $17(44)$ & 0.9 \\
\hline Previous smoker n (\%) & $54(55)$ & $22(56)$ & \\
\hline Prior MI n (\%) & $16(16)$ & $8(21)$ & 0.3 \\
\hline Prior $\mathrm{PCI}$ n $(\%)$ & $18(18)$ & $6(15)$ & 0.8 \\
\hline Prior CABG n (\%) & $5(5)$ & $2(5)$ & 0.9 \\
\hline STEMI $n(\%)$ & $40(41)$ & $19(49)$ & 0.6 \\
\hline LVEF \% & $52 \pm 9$ & $50 \pm 9$ & 0.2 \\
\hline \multicolumn{4}{|l|}{ Laboratory data at baseline } \\
\hline White blood cells $u \cdot \mu \mathrm{L}^{-1}$ & $10 \pm 3$ & $11 \pm 3$ & 0.7 \\
\hline Haemoglobin $\mathrm{g} \cdot \mathrm{dL}^{-1}$ & $13 \pm 2$ & $13 \pm 2$ & 0.6 \\
\hline Platelets $\mathrm{u} \cdot \mu \mathrm{L}^{-1}$ & 227 [189-266] & $223[192-280]$ & 0.6 \\
\hline \multicolumn{4}{|l|}{ Cardiovascular therapy $\mathrm{n}(\%)$} \\
\hline Aspirin & 97 (99) & $39(100)$ & 0.9 \\
\hline Ticagrelor & $55(56)$ & $20(52)$ & 0.6 \\
\hline Other P2Y12 inhibitor & $43(44)$ & $19(48)$ & \\
\hline Clopidogrel & $33(34)$ & $14(36)$ & 0.8 \\
\hline Prasugrel & $10(10)$ & $5(12)$ & 0.8 \\
\hline Beta-blocker & $84(86)$ & $35(90)$ & 0.8 \\
\hline ACE inhibitor/ARB & $88(90)$ & 37 (95) & 0.5 \\
\hline Statin & $96(98)$ & 37 (95) & 0.3 \\
\hline Calcium channel blockers & $5(5)$ & 2 (5) & 0.9 \\
\hline Nitrates & $2(2)$ & $1(2)$ & 0.9 \\
\hline Proton pump inhibitor ${ }^{\#}$ & $51(52)$ & $20(51)$ & 0.9 \\
\hline \multicolumn{4}{|l|}{ Biological parameters \% } \\
\hline Rate of apoptosis & $9.2 \pm 4$ & $13.2 \pm 3$ & $<0.001$ \\
\hline Patients receiving ticagrelor & $7.7 \pm 3$ & $12.2 \pm 2$ & $<0.001$ \\
\hline Patients receiving other $\mathrm{P} 2 \mathrm{Y} 12$ inhibitors & $11.1 \pm 5$ & $14.2 \pm 3$ & 0.0004 \\
\hline On-treatment PR after ADP stimulus & $32 \pm 14$ & $42 \pm 17$ & $<0.001$ \\
\hline Patients receiving ticagrelor & $24 \pm 11$ & $28 \pm 9$ & 0.3 \\
\hline Patients receiving other $\mathrm{P} 2 \mathrm{Y} 12$ inhibitors & $41 \pm 11$ & $57 \pm 7$ & $<0.001$ \\
\hline On-treatment PR after AA stimulus & $14 \pm 21$ & $22 \pm 20$ & $<0.001$ \\
\hline
\end{tabular}

Data are presented as mean $\pm S D$, unless otherwise stated." : pantoprazole was the only proton pump inhibitor administered. COPD: chronic obstructive pulmonary disease; UCOPD: undiagnosed/unknown COPD; BMI: body mass index; MI: myocardial infarction; PCl: percutaneous coronary intervention; CABG: coronary artery bypass graft; STEMI: ST-segment elevation MI; LVEF: left ventricular ejection fraction; ACE: angiotensin converting enzyme; ARB: angiotensin II receptor blocker; PR: platelet reactivity; ADP: adenosine diphosphate; $A A$ : arachidonic acid.

predictors of the rate of apoptosis. A two-sided value of $\mathrm{p}<0.05$ was considered significant. All analyses were performed with STATISTICA 8 (Statsoft Inc, Tulsa, OK, USA).

The study population included 137 patients [2]. At the 2-month spirometry, we found UCOPD in 39 (29\%) patients. A detailed description of all spirometric parameters has been previously reported [2]. Airflow limitation was mild in 23 (59\%) patients, moderate in 15 (38\%) patients and severe in one (3\%). Baseline characteristics of patients are summarised in table 1. Patients with UCOPD showed a significantly higher rate of apoptosis in HUVECs than those without COPD $(13.2 \pm 3 \%$ versus $9.2 \pm 4 \%$; $<<0.001)$. At multivariate analysis, UCOPD emerged as the independent predictor of the rate of apoptosis (HR 1.54, 95\% CI 1.3-1.8; $\mathrm{p}<0.001)$. In addition, we found a significant trend between the values of the rate of apoptosis and airflow limitation severity (mild $12.3 \pm 3$ versus moderate $14.3 \pm 2$ versus severe $18.7 ; \mathrm{p}=0.03$ ). On-treatment platelet reactivity was significantly higher in patients with UCOPD than others, both after ADP stimulus $(42 \pm 17 \%$ versus $32 \pm 14 \% ; \mathrm{p}<0.001)$ and arachidonic acid stimulus $(22 \pm 20 \%$ versus $14 \pm 21 \%$; $\mathrm{p}<0.001)$. We did not find any relation between on-treatment platelet reactivity and airflow limitation severity. Additionally, we analysed the effect of different P2Y12 inhibitors on endothelial dysfunction and 
platelet reactivity. Overall, ticagrelor was administered in 75 (55\%) patients (20, 52\% with UCOPD and 55, $56 \%$ without COPD). In patients treated with ticagrelor, the rate of apoptosis was lower than other P2Y12 inhibitors both in patients with UCOPD $(12.2 \pm 2 \%$ versus $14.2 \pm 3 \% ; \mathrm{p}=0.03)$ and in those without COPD $(7.7 \pm 3 \%$ versus $11.1 \pm 5 \%$; $<0.001)$. In addition, in patients with UCOPD, ticagrelor ensured ADP-induced platelet reactivity values similar to those observed in patients without COPD, (UCOPD $28 \pm 9 \%$ versus no COPD 24 $\pm 11 \%$; $=0.3$ ), while other P2Y12 inhibitors did not (UCOPD $57 \pm 7 \%$ versus no COPD $41 \pm 11 \%$; $\mathrm{p}<0.001)$.

The main aim of the SCAP trial was to validate a simple screening procedure to identify ACS patients with smoking history and at a risk of UCOPD. In the SCAP trial, according to current guidelines, spirometry was systematically applied to overpassing misdiagnosis and under diagnosis $[8,9]$. This sub-analysis highlighted that UCOPD is already associated with significant changes in endothelial function and on-treatment platelet reactivity. These physio-pathological elements may contribute to explain why $\mathrm{UCOPD}$ is related to poor prognosis in patients with ischaemic heart disease. We may speculate that these changes are reversible and an early identification of UCOPD may allow a timely cardiac and respiratory treatment with potentially beneficial prognosis.

For instance, we found that ticagrelor improved endothelial dysfunction, regardless of pulmonary function. We found that both COPD and UCOPD patients benefit from ticagrelor. A previous study on ACS patients showed that treatment with ticagrelor was able to improve endothelial function (tested with peripheral arterial tonometry) [10]. This was confirmed in a randomised clinical trial of COPD patients with stable coronary artery disease [6]. The present analysis confirms, in the subset of patients with UCOPD and ACS, this pleiotropic effect of ticagrelor on endothelial dysfunction, similarly for the relationship between ticagrelor and on-treatment platelet reactivity in UCOPD patients. It is well established that on-treatment platelet reactivity is heightened in COPD patients either with or without ischaemic heart disease [11]. This sub-study expands the observation showing that on-treatment platelet reactivity is heightened also in those with UCOPD. Ticagrelor modulates on-treatment platelet reactivity in ACS patients, regardless of the presence of UCOPD. This is not observed with other P2Y12 inhibitors. In the PLATelet inhibition and clinical Outcome (PLATO) trial, COPD patients showed greater benefit from ticagrelor treatment [12]. The combined effect of ticagrelor on endothelial function and on-treatment platelet reactivity helps to explain the mechanisms behind this observation. Similarly, we could extend, based on our findings, this potential benefit to UCOPD patients receiving ticagrelor.

In conclusion, to our best knowledge, this is the first study to show that patients with ACS and concomitant UCOPD have increased endothelial dysfunction and on-treatment platelet reactivity. Moreover, we showed that ticagrelor may contribute to improve both parameters. Accordingly, any further effort for early identification and treatment of patients with UCOPD should be encouraged.

Rita Pavasini $\odot^{1}$, Francesco Vieceli dalla Sega $^{2}$, Francesco Gallo ${ }^{1}$, Giulia Passarini ${ }^{1}$, Alberto Papi $\odot^{3}$, Marco Contoli ${ }^{3}$ and Gianluca Campo ${ }^{1,2,4}$

${ }^{1}$ Cardiovascular Institute, Azienda Ospedaliero-Universitaria di Ferrara, Cona, Italy. ${ }^{2}$ Laboratorio per le Tecnologie delle Terapie Avanzate (LTTA) Center, Ferrara, Italy. ${ }^{3}$ Research Centre on Asthma and COPD, Section of Internal and Cardio-Respiratory Medicine, Dept of Medical Sciences, University of Ferrara, Ferrara, Italy. ${ }^{4}$ Maria Cecilia Hospital, GVM Care and Research, E.S.: Health Science Foundation, Cotignola, Italy.

Correspondence: Rita Pavasini, Cardiovascular Institute, Azienda Ospedaliera Universitaria S. Anna, Via Aldo Moro 8, 44124, Cona (FE), Italy. E-mail: pvsrti@unife.it

Received: June 142017 | Accepted after revision: July 042017

This study is registered at ClinicalTrials.gov (number: NCT02324660).

Conflict of interest: None declared.

Acknowledgements: Author contributions are as follows. R. Pavasini is the guarantor of the content of the manuscript, including the data and analysis. R. Pavasini, G. Campo, F. Vieceli dalla Sega: conception, design, analysis and interpretation of data. R. Pavasini, G. Campo, M. Contoli, A. Papi: data analysis and interpretation. All authors: drafting of the manuscript and revising it critically for important intellectual content. All authors: final approval of the manuscript submitted.

\section{References}

1 Almagro P, Lapuente A, Pareja J, et al. Underdiagnosis and prognosis of chronic obstructive pulmonary disease after percutaneous coronary intervention: a prospective study. Int J Chron Obstruct Pulmon Dis 2015; 10: 1353-1361.

2 Campo G, Pavasini R, Barbetta C, et al. Predischarge screening for chronic obstructive pulmonary disease in patients with acute coronary syndrome and smoking history. Int J Cardio 2016; 222: 806-812.

3 Steg PG, James SK, Atar D, et al. ESC Guidelines for the management of acute myocardial infarction in patients presenting with ST-segment elevation. Eur Heart J 2012; 33: 2569-2619. 
4 Global Strategy for the Diagnosis, Management and Prevention of COPD, Global Initiative for Chronic Obstructive Lung Disease (GOLD) updated 2017. http://goldcopd.org/gold-2017-global-strategy-diagnosis-managementprevention-copd/ Date last updated: 2017. Date last accessed: June 1, 2017.

5 Valgimigli M, Agnoletti L, Curello S, et al. Serum from patients with acute coronary syndromes displays a proapoptotic effect on human endothelial cells: a possible link to pan-coronary syndromes. Circulation 2003; 107: 264-270.

6 Campo G, Vieceli Dalla Sega F, Pavasini R, et al. Biological effects of ticagrelor over clopidogrel in patients with stable coronary artery disease and chronic obstructive pulmonary disease. Thromb Haemost 2017; 117: 1208-1216.

7 Campo G, Parrinello G, Ferraresi P, et al. Prospective evaluation of on clopidogrel platelet reactivity over time in patients treated with percutaneous coronary intervention relationship with gene polymorphisms and clinical outcome. J Am Coll Cardiol 2011; 57: 2474-2483.

8 Fernández-Villar A, Soriano JB, López-Campos JL. Overdiagnosis of COPD: precise definitions and proposals for improvement. Br J Gen Pract 2017; 67: 183-184.

9 Franssen FM, Soriano JB, Roche N, et al. Lung function abnormalities in smokers with ischemic heart disease. Am J Respir Crit Care Med 2016; 194: 568-576.

10 Torngren K, Ohman J, Salmi H, et al. Ticagrelor improves peripheral arterial function in patients with a previous acute coronary syndrome. Cardiology 2013; 124: 252-258.

11 Campo G, Pavasini R, Pollina A, et al. On-treatment platelet reactivity in patients with chronic obstructive pulmonary disease undergoing percutaneous coronary intervention. Thorax 2014; 69: 80-81.

12 Andell P, James SK, Cannon CP, et al. Ticagrelor versus clopidogrel in patients with acute coronary syndromes and chronic obstructive pulmonary disease: an analysis from the Platelet Inhibition and Patient Outcomes (PLATO) trial. J Am Heart Assoc 2015; 4: e002490. 